\title{
High strain rate thermoplastic demolding of metallic glasses
}

\author{
Molla Hasan, Golden Kumar* \\ Department of Mechanical Engineering, Texas Tech University, Lubbock, TX 79409, USA
}

\begin{abstract}
Silicon templates are ideal for thermoplastic patterning of metallic glasses but their thermal expansion mismatch prevents demolding without chemical etching. Besides limiting the template life, chemical exposure alters the surface chemistry and properties of metallic glasses. To overcome these issues, we develop thermoplastic demolding which enables template reusability and fabrication of pristine metallic glass structures. Chemical-free demolding allows decoupling of topographic and compositional effects on properties of metallic glasses. We show that mechanically and chemically demolded samples exhibit distinct wetting behavior despite similar topography. The versatility of demolding technique is demonstrated for structures of different shapes and metallic glass formers.
\end{abstract}

*Corresponding author:

Dr. Golden Kumar

Email:golden.kumar@ttu.edu

Tel: 806-834-8123 
Patterning is an effective route to change the surface properties such as adhesion [1-4], cell-response $[5,6]$, friction $[7,8]$, reflectance $[9,10]$, and wetting $[3,11,12]$. Numerous techniques such as lithography [13-15], embossing [16-18], self-assembly [19, 20], electrochemical etching [21], and laser processing [22] have been developed for patterning of semiconductors and polymers. These methods are either not readily applicable to metals or require complex hardware. Metallic glasses (MGs) are unique alloys of metals which can be patterned by simple thermoplastic methods due to the existence of their supercooled liquid state [23-27]. This is achieved by molding of MGs against templates heated above the glass transition temperature $\left(T_{g}\right)$ followed by cooling below $T_{g}$ and demolding [27]. MG surfaces textured with micro [28-30], nano [31-33], and hierarchical [34] structures have been synthesized by using suitable templates. Structures harvested from the patterned surfaces have also been used for characterization of size-effects in MGs [35]. However, in all these cases the templates are chemically etched away to release the MGs. Use of chemicals not only limits the template reusability but can also affect the properties of molded MGs. It has been reported that $\mathrm{KOH}$ typically used for wet etching of $\mathrm{Si}$ and Alumina templates can de-alloy some Zr-based MGs [36]. $\mathrm{KOH}$ is known to decrease the hydrophobicity of materials by altering the surface chemistry and topography [37]. Size-effects are also very sensitive to the processing conditions in MGs due to their metastable structure [38]. Therefore, chemical-free demolding of templates is essential to use thermoplastic fabrication for surface engineering and characterization of small structures in MGs.

Mechanical separation of templates and MGs after thermoplastic molding is prevented by their thermal expansion mismatch (Figure 1). The coefficient of thermal expansion $(\alpha)$ for MG formers is often higher than templates (Table 1). But any combination of mismatch in $\alpha$ ( $\alpha_{\mathrm{MG}}>$ 
$\alpha_{\text {template }}$ or $\left.\alpha_{\mathrm{MG}}<\alpha_{\text {template }}\right)$ results in thermal stress build up on the multiple MG-template interfaces during cooling below $T_{g}$ (Figure 1a). To estimate the extent of residual thermal stress, a 2D thermomechanical analysis was performed using finite element method for the Pt-based $\left(\mathrm{Pt}_{57.3} \mathrm{Cu}_{14.6} \mathrm{Ni}_{5.3} \mathrm{P}_{22.8}\right) \mathrm{MG}$ molded against silicon ( $\mathrm{Si}$ ) template (Figure $1 \mathrm{~b}$ ). Two rectangular $\mathrm{Si}$ cavities filled with Pt-based MG were cooled from $503 \mathrm{~K}$ ( $T_{g}$ of Pt-based $\mathrm{MG}$ ) to room temperature. The thermal stress distribution due to mismatch in $\alpha$ was calculated using the elastic constants of Pt-based MG and Si. The residual stress in thermoplastic operations develops only during cooling below $T_{g}$ above which the MG supercooled liquid can relax through fast structural rearrangement. Compressive stress as high as $177 \mathrm{MPa}$ was observed on the interface due to different thermal shrinkage of Pt-based MG and Si (Figure 1b). The stress is highest at the interface close to the MG substrate due to its large shrinkage. Similar analysis was performed for other MGs and Si templates and the resulting values of maximum thermal stress are listed in Table 1. This residual stress must be minimized for characterization of intrinsic mechanical behavior of MG structures prepared by thermoplastic molding.

Thus, there are three primary reasons why an alternative demolding method for thermoplastically formed MGs is required: (1) to produce chemical-free MG patterns for understanding the topographic effects, (2) to fabricate stress-free MG structures for mechanical testing, and (3) to enable reusability of expensive lithographic templates. In this paper, we describe a novel high temperature mechanical demolding of MGs by utilizing the strain rate effects. We show that thermoplastic molding and demolding of a MG supercooled liquid can be performed at the same temperature but varying strain rates without sacrificing the template or the MG features. 
MG supercooled liquids exhibit a variety of flow behaviors depending on the temperature (viscosity) and strain rate (Figure 2a). At low strain rates, the MG supercooled liquids flow homogeneously as Newtonian fluids [39-42]. At higher strain rates the viscosity of MG supercooled liquids becomes strain rate dependent and the flow becomes non-Newtonian but remains spatially homogeneous $[40,43]$. Further increase in strain rate results in solid-like yielding and inhomogeneous (shear-localized) deformation in MG supercooled liquids [40, 41]. Low strain rates are used for thermoplastic molding because the Newtonian behavior of MG supercooled liquids can be precisely described using constitutive equations to model the shaping process $[44,45]$. In addition, low strain rate loading minimizes the risk of premature template failure. Ideally, the demolding should be performed above $T_{g}$ to avoid the thermal stress but it results in distortion of MG structures because their flow stress (cohesive strength) is lower than the adhesive strength. The flow stress of MG supercooled liquids increases with increasing strain rate in Newtonian and non-Newtonian regimes and becomes comparable to the room temperature yield strength in the shear-localized regime [40]. We propose to utilize this strain rate dependent strengthening of MG supercooled liquids for thermoplastic demolding (Figure 2b). Thermoplastic molding is performed at a low strain rate whereas the demolding is performed at a higher strain rate but the same temperature. Above a critical strain rate, the flow stress of MG supercooled liquid exceeds its adhesive strength resulting in failure at the MG-template interface. Isothermal processing overcomes the issue of thermal stress while different strain rates invoke cohesive and adhesive failure in MG supercooled liquids during molding and demolding, respectively (Figure $2 b$ ). 
Figure 3 demonstrates the feasibility of proposed thermoplastic demolding of MGs. Figures $3 \mathrm{a}$ and $3 \mathrm{~b}$ compare the SEM images of patterned MG (Pt-based) released by chemical and mechanical demolding, respectively. The chemically demolded sample was prepared by molding against a $\mathrm{Si}$ template at $270^{\circ} \mathrm{C}$ followed by etching of $\mathrm{Si}$ in $\mathrm{KOH}$ at room temperature. The mechanically demolded sample was prepared by slow pressing $(\sim 0.02 \mathrm{~mm} / \mathrm{s})$ and fast pulling $(\sim 12 \mathrm{~mm} / \mathrm{s})$ at $270^{\circ} \mathrm{C}$ using a Si template. Similarity between the overall geometry of pattern features in the chemically and mechanically demolded samples demonstrates the capability of high strain rate mechanical separation. The SEM image of Si template after mechanical demolding shows that the template is free of cracks and MG residue (Figure 3c). Therefore, the demolded template can be reused multiple times to enable cost-effective patterning of MGs. Although the critical strain rate for demolding is determined by the feature dimensions and the type of MG former, but a strain rate higher than $10^{2} \mathrm{~s}^{-1}$ was sufficient to demold three $\mathrm{MG}$ formers considered here: Pd-based $\left(\mathrm{Pd}_{43} \mathrm{Cu}_{27} \mathrm{Ni}_{10} \mathrm{P}_{20}\right), \quad \mathrm{Zr}$-based $\left(\mathrm{Zr}_{35} \mathrm{Ti}_{30} \mathrm{Cu}_{8.25} \mathrm{Be}_{26.75}\right)$, and Pt-based. Square micro-pillars were mechanically demolded after thermoplastic embossing of three MG formers on Si templates (Figures 3d-f). Thermoplastic molding of MGs has been extensively studied but nondestructive demolding is reported for the first time to the best of our knowledge.

It is of fundamental interest to study how the topography affects the surface properties of materials. Progress in thermoplastic patterning of MGs has motivated recent studies on wetting [34, 46, 47], cell-adhesion [48], and reflectance [49] of textured MGs. Lithographically fabricated $\mathrm{Si}$ and/or anodized porous Alumina are used as templates to produce a systematic variation in topography of MGs for such studies. However, the use of chemicals such as $\mathrm{KOH}$ to dissolve the templates can alter the surface chemistry of patterned MGs and can convolute the 
topographic effects. Figure 4 shows the effects of surface chemistry and topography on the wetting behavior of Pt-based MG. The water contact angle (CA) increases from $55^{\circ}$ to $113^{\circ}$ with varying surface texture in mechanically demolded samples. Therefore, Cassie-Baxter condition of solid-air-water interface for hydrophobicity can be stabilized in intrinsically hydrophilic MGs through topographic engineering. In contrast, all samples exposed to $\mathrm{KOH}$ exhibit hydrophilic $\left(\mathrm{CA} \sim 25-38^{\circ}\right)$ behavior despite large variation in topography. These results suggest that use of $\mathrm{KOH}$ for chemical demolding obscures the topographic effects on wetting behavior of MGs. The mechanically demolded MG structures are also free of thermal stress because they are cooled after separation from the templates. Therefore, mechanical demolding presented here provides an ideal platform to fabricate pristine MG structures for characterization of surface and mechanical properties.

In summary, we have demonstrated that templates can be nondestructively demolded after thermoplastic molding of MGs by optimizing the strain rate. Molding and demolding are performed isothermally in the supercooled liquid state under different strain rates to overcome the issues of thermal stress and no-slip at MG-template interface. Demolding was successfully applied to three different MG formers and a wide range of pattern geometries. Nondestructive demolding and reusability of templates provide low-cost patterning technique for MGs. We show that demolding technique strongly affects the properties of MG structures and use of chemical has deleterious effects. Mechanical demolding generates chemical-free MG structures which can be utilized to decouple the topographic and chemical effects on the surface properties of MGs. 


\section{Acknowledgement}

The work was supported by the National Science Foundation through CMMI-1266277. The

SEM characterization was conducted using Hitachi S-4300 acquired through NSF Major

Research Instrumentation Program Award \#0421032.

\section{References}

[1]. A. K. Geim, S. V. Dubonos, I. V. Grigorieva, K. S. Novoselov, A. A. Zhukov and S. Y. Shapoval, Nat. Mater. 2 (2003) 461.

[2]. $\quad$ H. Lee, B. P. Lee and P. B. Messersmith, Nature 448 (2007) 338.

[3]. B. Bhushan and Y. C. Jung, Prog. Mater. Sci. 56 (2011) 1.

[4]. $\quad$ B. Aksak, M. P. Murphy and M. Sitti, Langmuir 23 (2007) 3322.

[5]. J. Y. Lim and H. J. Donahue, Tissue Eng. 13 (2007) 1879.

[6]. R. Singhvi, A. Kumar, G. P. Lopez, G. N. Stephanopoulos, D. I. C. Wang, G. M. Whitesides and D. E. Ingber, Science 264 (1994) 696.

[7]. $\quad$ Y. C. Jung and B. Bhushan, Nanotechnology 17 (2006) 4970.

[8]. M. Wakuda, Y. Yamauchi, S. Kanzaki and Y. Yasuda, Wear 254 (2003) 356.

[9]. Z. P. Yang, L. J. Ci, J. A. Bur, S. Y. Lin and P. M. Ajayan, Nano Lett. 8 (2008) 446.

[10]. V. V. Iyengar, B. K. Nayak and M. C. Gupta, Appl. Opt. 49 (2010) 5983.

[11]. J. Bico, U. Thiele and D. Quere, Colloids Surf. A 206 (2002) 41.

[12]. M. E. Abdelsalam, P. N. Bartlett, T. Kelf and J. Baumberg, Langmuir 21 (2005) 1753.

[13]. Y. N. Xia and G. M. Whitesides, Annu. Rev. Mater. Sci. 28 (1998) 153.

[14]. S. Y. Chou, P. R. Krauss and P. J. Renstrom, Science 272 (1996) 85.

[15]. K. Y. Suh, Y. S. Kim and H. H. Lee, Adv. Mater. 13 (2001) 1386.

[16]. Y. N. Xia, J. A. Rogers, K. E. Paul and G. M. Whitesides, Chem. Rev. 99 (1999) 1823.

[17]. B. D. Gates, Q. B. Xu, M. Stewart, D. Ryan, C. G. Willson and G. M. Whitesides, Chem. Rev. 105 (2005) 1171.

[18]. M. Heckele and W. K. Schomburg, J. Micromech. Microeng. 14 (2004) R1.

[19]. N. L. Abbott, J. P. Folkers and G. M. Whitesides, Science 257 (1992) 1380.

[20]. R. K. Smith, P. A. Lewis and P. S. Weiss, Prog. Surf. Sci. 75 (2004) 1.

[21]. S. E. Skrabalak, J. Y. Chen, Y. G. Sun, X. M. Lu, L. Au, C. M. Cobley and Y. N. Xia, Acc. Chem. Res. 41 (2008) 1587.

[22]. A. Y. Vorobyev and C. L. Guo, Laser Photonics Rev. 7 (2013) 385.

[23]. W. L. Johnson, MRS Bull. 24 (1999) 42.

[24]. A. Inoue, Acta Mater. 48 (2000) 279.

[25]. A. L. Greer, Mater. Today 12 (2009) 14.

[26]. W. H. Wang, C. Dong and C. H. Shek, Mater. Sci. Eng., R 44 (2004) 45.

[27]. J. Schroers, Adv. Mater. 22 (2010) 1566.

[28]. Y. Saotome, K. Imai, S. Shioda, S. Shimizu, T. Zhang and A. Inoue, Intermetallics 10 (2002) 1241.

[29]. G. Kumar, A. Desai and J. Schroers, Adv. Mater. 23 (2011) 461. 
[30]. D. L. Henann, V. Srivastava, H. K. Taylor, M. R. Hale, D. E. Hardt and L. Anand, J. Micromec. Microeng. 19 (2009) 115030.

[31]. Y. Saotome, K. Itoh, T. Zhang and A. Inoue, Scr. Mater. 44 (2001) 1541.

[32]. G. Kumar, H. X. Tang and J. Schroers, Nature 457 (2009) 868.

[33]. J. P. Chu, H. Wijaya, C. W. Wu, T. R. Tsai, C. S. Wei, T. G. Nieh and J. Wadsworth, Appl. Phys. Lett. 90 (2007) 034101.

[34]. M. Hasan, J. Schroers and G. Kumar, Nano Lett. 15 (2015) 963.

[35]. D.J. Magagnosc, G. Kumar, J. Schroers, P. Felfer, J.M. Cairney, and D.S. Gianola, Acta Mater. 74 (2014) 165.

[36]. M. Hasan, N. Kahler and G. Kumar, ACS Appl. Mater. Inter. 8 (2016) 11084.

[37]. D. Maji, S. K. Lahiri and D. Das, Surf. Interface Anal. 44 (2012) 62.

[38]. J. Yi, W.H. Wang and J. Lewandowski, Acta Mater. 87 (2015) 1.

[39]. F. Spaepen, Acta Metall. Mater. 25 (1977) 407.

[40]. J. Lu, G. Ravichandran and W. L. Johnson, Acta Mater. 51 (2003) 3429.

[41]. A. H. Vormelker, O. L. Vatamanu, L. Kecskes and J. J. Lewandowski, Metall. Mater.Trans. 39A (2008) 1922.

[42]. S. X. Song, J. S. C. Jang, J. C. Huang and T. G. Nieh, Intermetallics 18 (2010) 702.

[43]. Y. Kawamura, T. Shibata, A. Inoue and T. Masumoto, Appl. Phys. Lett. 69 (1996) 1208.

[44]. G. Kumar, J. Schroers and J. Blawzdziewicz, Nanotechnology 24 (2013) 105301.

[45]. J. Ma, L. S. Huo, D. Q. Zhao and W. H. Wang, J. Appl. Phys. 113 (2013) 104505.

[46]. K. S. Liu, Z. Li, W. H. Wang and L. Jiang, Appl. Phys. Lett. 99 (2011) 261905.

[47]. A. S. Arora, Q. Xu, Z. Xia, Y. H. Ho, N. Dahotre, J. Schroers and S. Mukherjee, Scr. Mater. 69 (2013) 732.

[48]. J. Padmanabhan, E. R. Kinser, M. A. Stalter, C. Duncan-Lewis, J. L. Balestrini, A. J. Sawyer, J. Schroers and T. R. Kyriakides, ACS Nano 8 (2014) 4366.

[49]. H. Tarigan, N. Kahler, N. Ramos, G. Kumar and A. Bernussi, Appl. Phys. Lett. 107 (2015) 021903.

[50] . W. H. Wang, Prog. Mater. Sci. 57 (2012) 487.

[51]. H. Watanabe, N. Yamada and M. Okaji, Int. J. Thermodyn. 25 (2004) 221.

[52]. X. R. Zhang, T. S. Fisher, A. Raman and T.D. Sands, Nanoscale Microscale Thermophys. Eng. 13 (2009) 243.

[53]. J. Y. Suh, Ph.D. Thesis, Caltech (2009).

[54]. Y. He, R. B. Schwarz, D. Mandrus and L. Jacobson, J. Non-Cryst. Solids 207 (1996) 602.

[55]. H. Kato, H. S. Chen and A. Inoue, Scr. Mater. 58 (2008) 1106. 

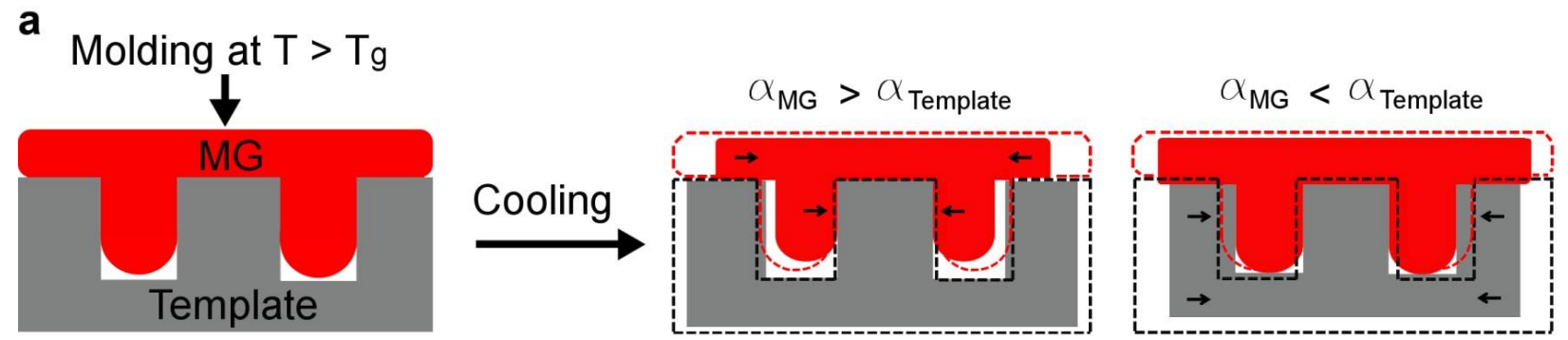

b
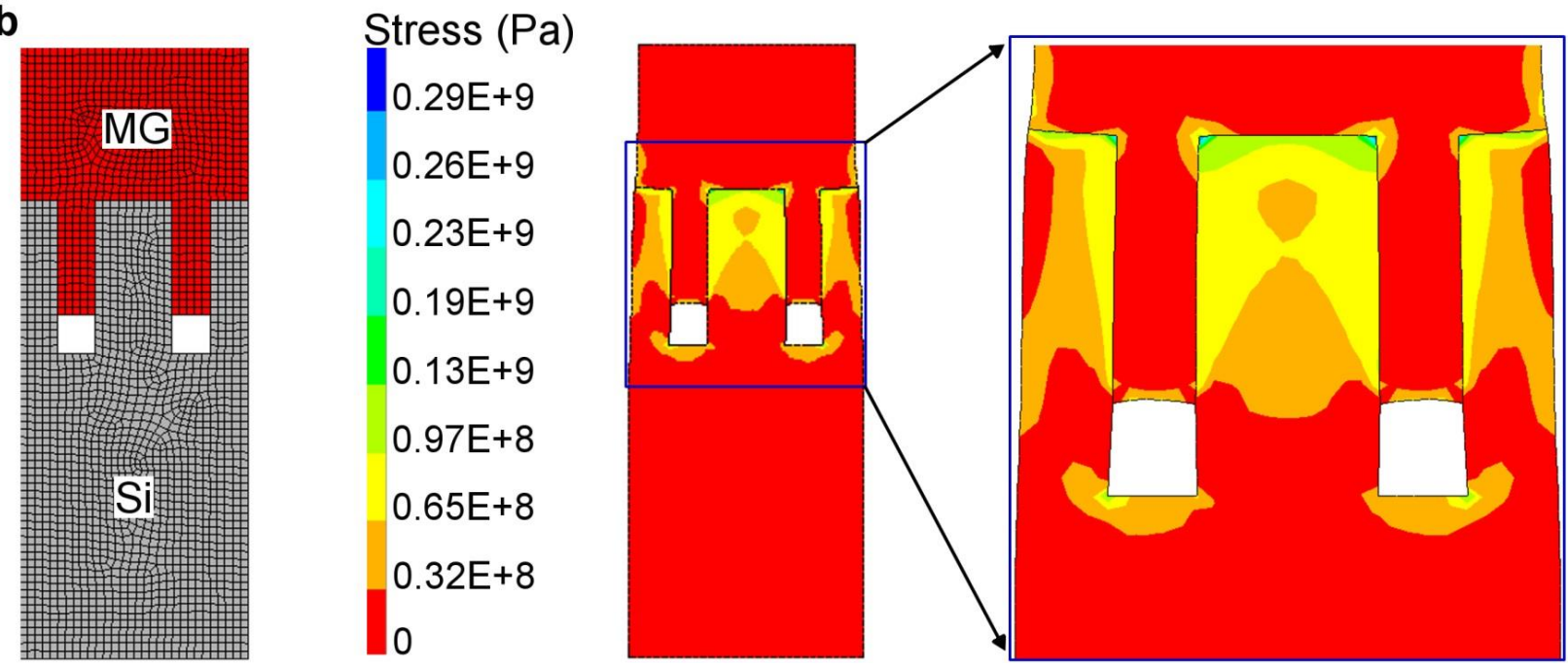

Figure 1: Thermal stress development during thermoplastic molding of MGs. The schematic (a) illustrates that thermal stress is generated for any combination of mismatch $\left(\alpha_{\mathrm{MG}}>\alpha_{\text {Template }}\right.$ or $\left.\alpha_{\mathrm{MG}}<\alpha_{\text {Template }}\right)$ in thermal expansion coefficients. Thermomechanical analysis for $\mathrm{Si}$ and Ptbased MG preformed using finite element method shows the distribution in thermal stress at MG-template interface (b). 


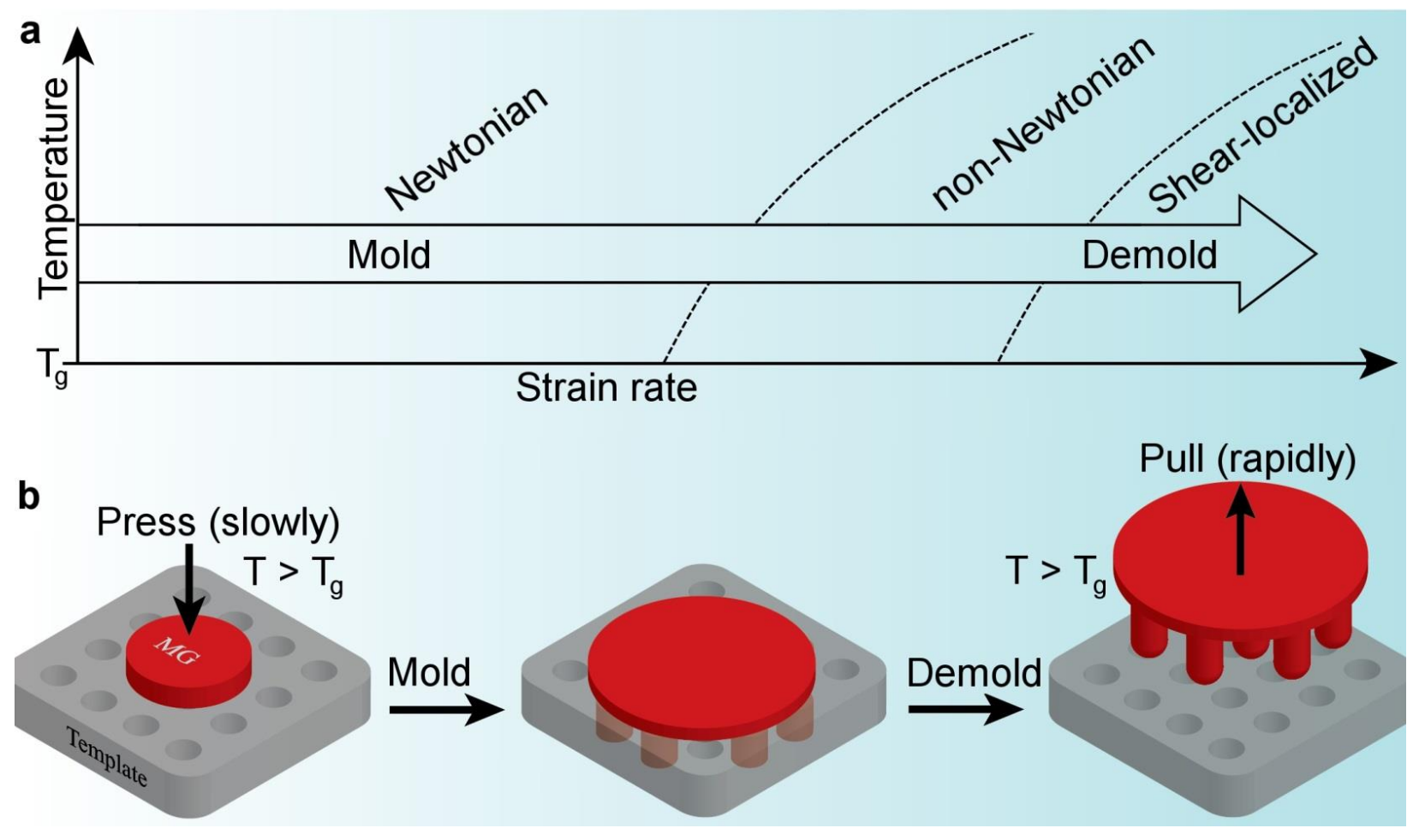

Figure 2: Using the effect of strain rate for molding and demolding operations with MG supercooled liquids. Schematic illustration of different flow regimes (Newtonian, nonNewtonian, and shear-localized) observed in MG supercooled liquids (a). The proposed approach for thermoplastic demolding of MGs (b). MG is molded at a low strain rate followed by high strain rate demolding at the same temperature. Isothermal processing eliminates the thermal stress whilst high strain rate demolding promotes adhesive failure. 
a: chemically demolded

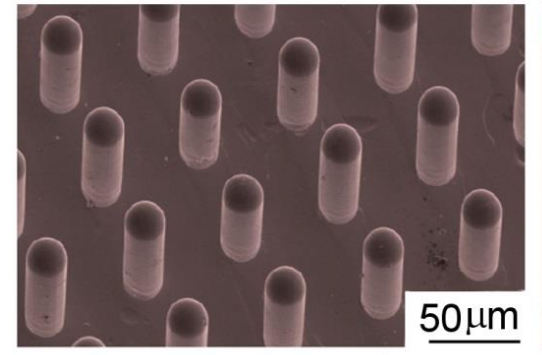

\section{d: Pd-based}

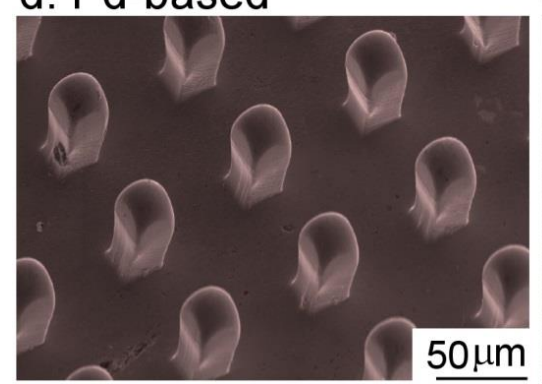

b: mechanically demolded c: Si template after use
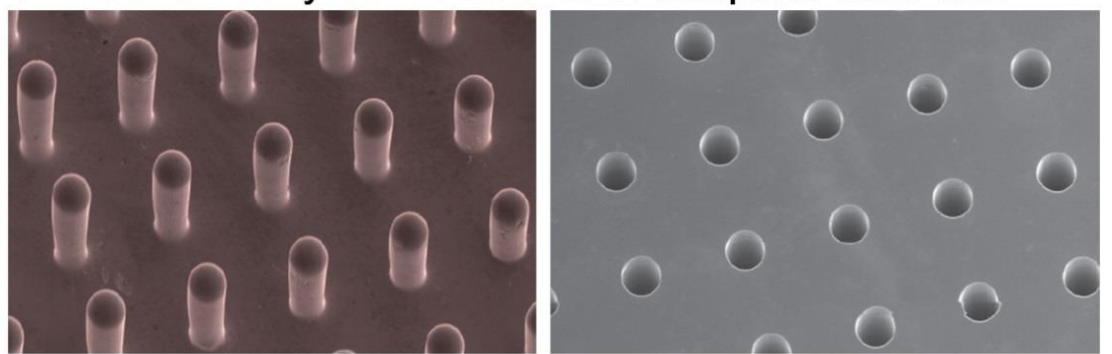

e: Zr-based

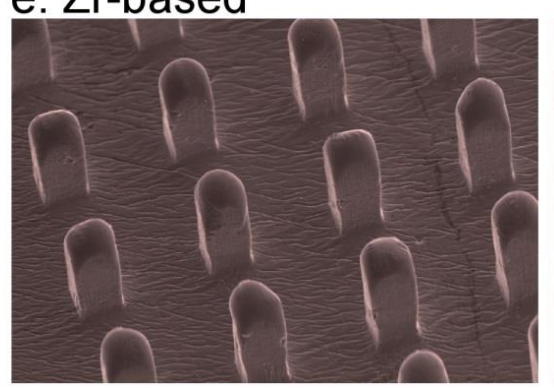

f: Pt-based

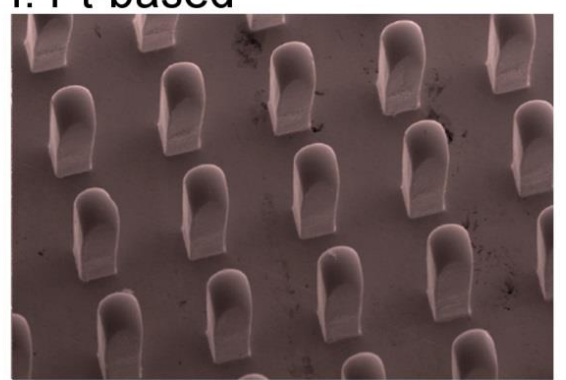

Figure 3: Feasibility and versatility of proposed mechanical demolding of thermoplastically formed MGs. Comparison of Pt-based MG microstructures released by chemical etching (a) and mechanical demolding (b) of Si templates. SEM image of a Si template after mechanical demolding showing its reusability (c). SEM images of mechanically demolded microstructures for different MG formers: Pd-based (d), Zr-based (e), and Pt-based (f). 


\section{Topography Mechanically demolded After $\mathrm{KOH}$ exposure}

\section{a: flat}

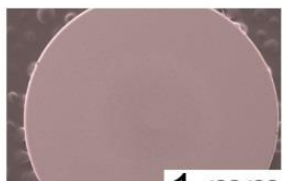

$1 \mathrm{~mm}$
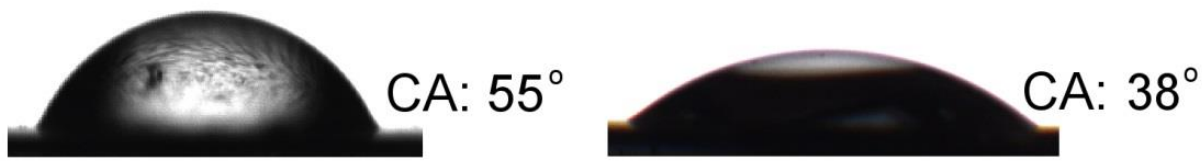

b: micro-pillars
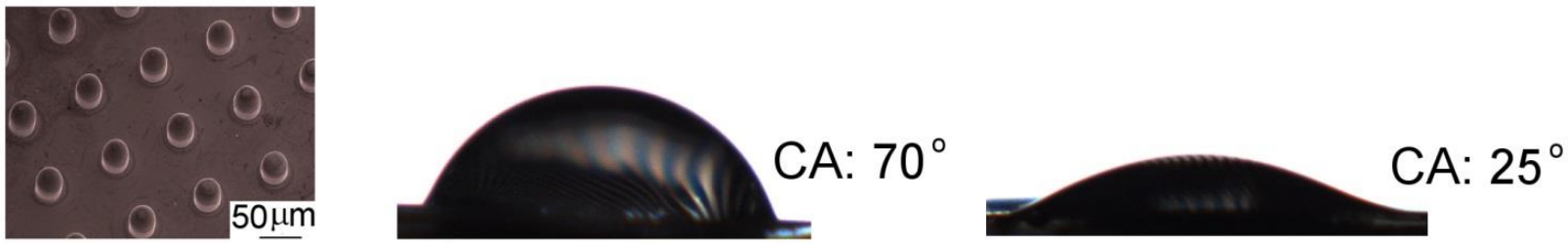

c: micro-tubes
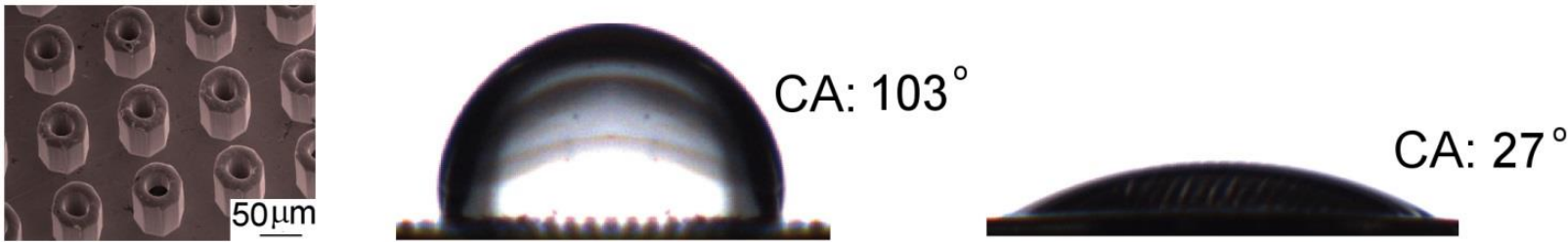

$\mathrm{d}$ : honeycomb
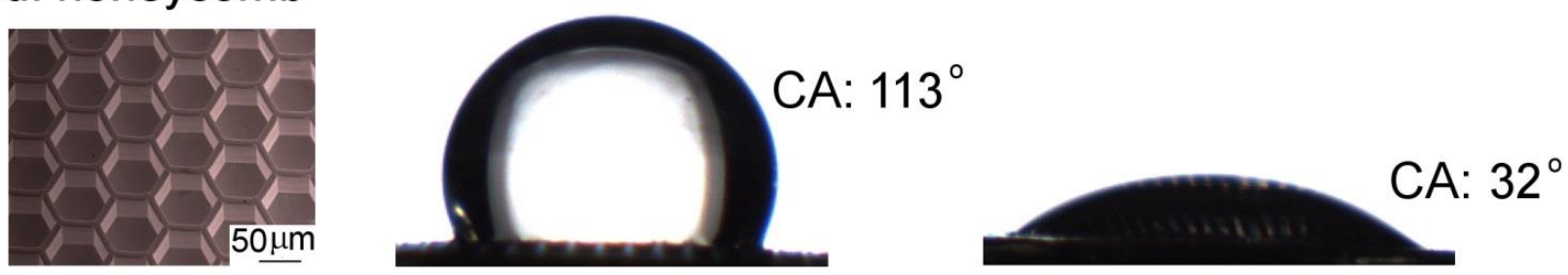

Figure 4: Effect of surface chemistry and topography on the wetting behavior of Pt-based MG. The results show that mechanically demolded samples show a clear correlation between the CA and surface topography. In contrast, $\mathrm{KOH}$ exposure $(5 \mathrm{~min})$ obscures the topographic effects and renders all samples hydrophilic. The measurement error in CA is about $\pm 5^{\circ}$. 
Table 1: The $\alpha, T_{g}$, and Young's modulus for selected templates and $\mathrm{MG}$ formers. The maximum thermal stress is calculated from the thermomechanical analysis of MG formers cooled in Silicon from the $T_{g}$ to room temperature. The elastic constants of MGs are taken from Ref. [50].

\begin{tabular}{|c|c|c|c|c|c|}
\hline & Material & $\alpha\left(\times 10^{-6} \mathrm{~K}^{-1}\right)$ & $T_{g}(\mathbf{K})$ & $\begin{array}{c}\text { Young's } \\
\text { modulus } \\
(\text { GPa) }\end{array}$ & $\begin{array}{c}\text { Maximum } \\
\text { thermal stress } \\
\text { (MPa) }\end{array}$ \\
\hline \multirow[t]{3}{*}{ Templates } & Silicon & $2.8[51]$ & & 169 & \\
\hline & Nanoporous Alumina & $16.7[52]$ & & $100-125$ & \\
\hline & Electroplated Nickel & 13 & & $165-205$ & \\
\hline \multirow[t]{5}{*}{ MGs } & $\mathrm{Pt}_{57.5} \mathrm{Cu}_{14.7} \mathrm{Ni}_{5.3} \mathrm{P}_{22.5}$ & $12.5[53]$ & 503 & 94.8 & 177 \\
\hline & $\mathrm{Pd}_{43} \mathrm{Cu}_{27} \mathrm{Ni}_{10} \mathrm{P}_{20}$ & $14.1[53]$ & 588 & 93.4 & 289 \\
\hline & $\mathrm{Z}_{35} \mathrm{Ti}_{30} \mathrm{Cu}_{8.25} \mathrm{Be}_{26.75}$ & $9.3[53]$ & 578 & 86.9 & 155 \\
\hline & $\mathrm{Zr}_{41.2} \mathrm{Ti}_{13.8} \mathrm{Cu}_{12.5} \mathrm{Ni}_{10} \mathrm{Be}_{22.5}$ & $9.9[54]$ & 623 & 96 & 205 \\
\hline & $\mathrm{Zr}_{65} \mathrm{Al}_{7.5} \mathrm{Cu}_{17.5} \mathrm{Ni}_{10}$ & $11.3[55]$ & 653 & 82 & 252 \\
\hline
\end{tabular}




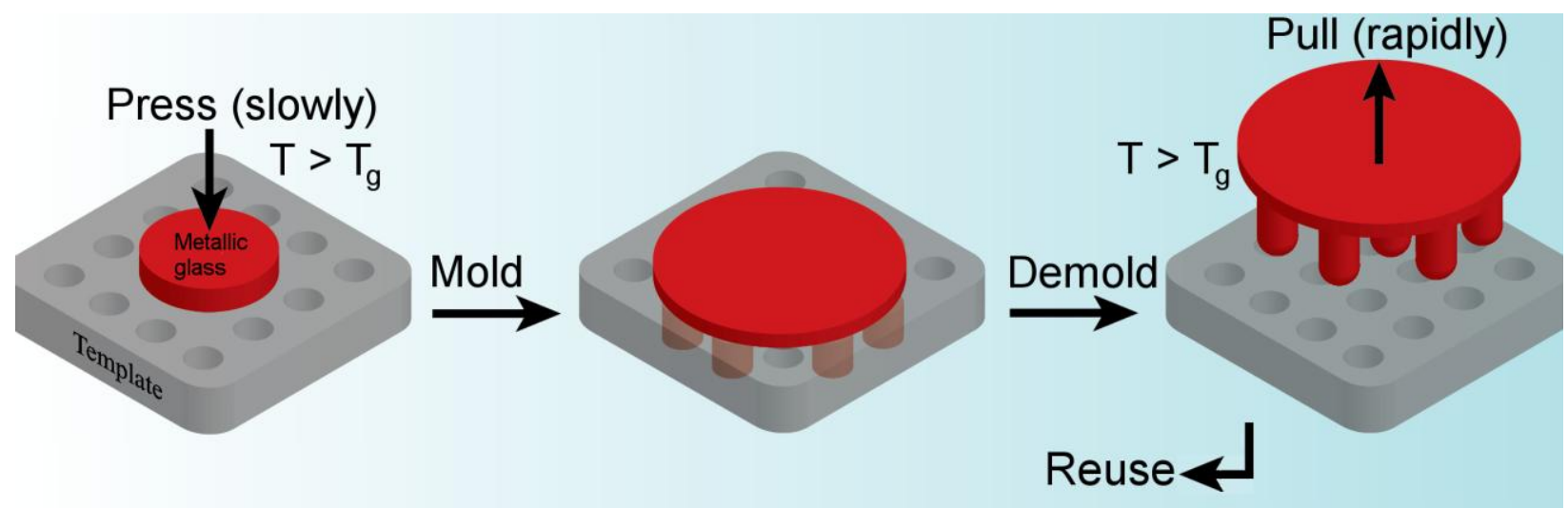

